\title{
Applied Research on Signal Processing Deducing by Method of Completing the Squares of Least Square Estimation
}

\author{
ZHANG Eryan \\ Department of Basic Courses \\ Beijing Institute of Graphic Communication \\ Beijing, China \\ zhanger-yan1@bigc.edu.cn
}

\author{
GAO Linlin \\ Press and Publication Institute \\ Beijing Institute of Graphic Communication \\ Beijing, China \\ 865835170@qq.com
}

\begin{abstract}
The method of completing the squares is a method that is used to transform a math formula directionally in order to obtain a perfect square formula by making rational use of "splitting items" and "adding items”. Least square estimation consists of the method of projection, the method of derivative and the method of completing the squares. By comparing and analyzing the three methods, the conclusion has been drawn that the method of completing the squares is the best, as it provides the necessary conditions in which the objective function can reduce to minimum. Such methods as solving Wiener filtering and system identification have been taken as examples to illustrate that not only optimal estimation but also the extreme value of the objective function can be obtained if the method of completing the squares is applied rationally into solving problems of least squares.
\end{abstract}

Keywords- least square method; method of completing the squares; Wiener filtering; system identification ; application

\section{INTRODUCTION}

Least square estimation consists of the method of projection, the method of derivative and the method of completing the squares. The method of completing the squares is a method that is used to transform a math formula directionally in order to obtain a perfect square formula by making rational use of "splitting items" and "adding items". Theoretical basis for this approach is completely square formula, sometimes we call it "patchwork with squares method". We use reasonable method of completing the squares to solve the least squares problem, not only to determine the best estimate, but also at the same time draw extreme value of the objective function[]1-3]. This paper discusses the method of least squares method of completing the squares Wiener filter applications and system identification problems.

\section{THE THREE MANIFESTATIONS OF LEAST-SQUARES METHOD}

Generally, the method for solving linear approximation in Hilbert space is called the Least square method. Least square estimation consists of the method of projection, the method of derivative and the method of completing the squares. In practice, they each have their own advantages and disadvantages in different situations depending on the needs and possibilities, the flexibility to choose and use appropriate methods, is the key to master the least squares method.

Below to Fourier series expansion to be explained these three forms[4].

Resuming $\left\{\boldsymbol{e}_{1}, \boldsymbol{e}_{2}, \cdots\right\}$ is a set of orthogonal normalized elements of Hilbert space, $\boldsymbol{x}$ is an element of the Hilbert space.

The least squares method is to find $\boldsymbol{m}_{0} \in \boldsymbol{M}$ to meet need of $\left\|\boldsymbol{x}-\boldsymbol{m}_{0}\right\|=\min _{\boldsymbol{m} \in \boldsymbol{M}}\|\boldsymbol{x}-\boldsymbol{m}\|$

in formed by the sub-basement space of $M=\operatorname{span}\left\{\boldsymbol{e}_{1}, \boldsymbol{e}_{2}, \cdots\right\}$.

\section{A. The Method of Projection}

We know from the projection theorem optimal coefficients should meet the condition: $\left(x-\sum_{i=1}^{\infty} \boldsymbol{k}_{i} \boldsymbol{e}_{i}\right) \perp \boldsymbol{e}_{\boldsymbol{m}}, \boldsymbol{m}=1,2, \cdots$

Then,

$$
\begin{aligned}
& \left(\boldsymbol{x}, \boldsymbol{e}_{\boldsymbol{m}}\right)=\left(\sum_{\boldsymbol{i}=1}^{\infty} \boldsymbol{k}_{\boldsymbol{i}} \boldsymbol{e}_{\boldsymbol{i}}, \boldsymbol{e}_{\boldsymbol{m}}\right)=\boldsymbol{k}_{\boldsymbol{m}} \text {, if and only if } \\
& \boldsymbol{k}_{\boldsymbol{i}}=\boldsymbol{c}_{\boldsymbol{i}} .
\end{aligned}
$$


$\boldsymbol{Q}\left(\boldsymbol{k}_{1}, \boldsymbol{k}_{2}, \cdots\right)$ is achieved the minimum, here $\boldsymbol{c}_{\boldsymbol{i}}=\left(\boldsymbol{x}, \boldsymbol{e}_{\mathrm{i}}\right), \quad \boldsymbol{i}=1,2, \cdots$.

\section{B. The Method of Derivative}

Resuming function

$$
\begin{aligned}
& \boldsymbol{Q}\left(\boldsymbol{k}_{1}, \boldsymbol{k}_{2}, \cdots\right) \\
= & \left\|\boldsymbol{x}-\sum_{\boldsymbol{i}=1}^{\infty} \boldsymbol{k}_{\boldsymbol{i}} \boldsymbol{e}_{\boldsymbol{i}}\right\|^{2} \\
= & \left(\boldsymbol{x}-\sum_{\boldsymbol{i}=1}^{\infty} \boldsymbol{k}_{\boldsymbol{i}} \boldsymbol{e}_{\boldsymbol{i}}, \boldsymbol{x}-\sum_{\boldsymbol{j}=1}^{\infty} \boldsymbol{k}_{\boldsymbol{j}} \boldsymbol{e}_{\boldsymbol{j}}\right) \\
= & \|\boldsymbol{x}\|^{2}-2 \sum_{\boldsymbol{i}=1}^{\infty} \boldsymbol{k}_{\boldsymbol{i}} \boldsymbol{c}_{\boldsymbol{i}}+\sum_{\boldsymbol{i}=1}^{\infty} \boldsymbol{k}_{\boldsymbol{i}}{ }^{2} .
\end{aligned}
$$

The method of derivative is to use derivatives to seek the optimal coefficients $\boldsymbol{k}_{1}, \boldsymbol{k}_{2}, \cdots$ so as to satisfy the condition: $\frac{\partial \boldsymbol{Q}}{\partial \boldsymbol{k}_{\boldsymbol{i}}}=0, \quad i=1,2, \cdots$.

After solving the above system of equations, we get such a result as $-2 \boldsymbol{c}_{\boldsymbol{i}}+2 \boldsymbol{k}_{\boldsymbol{i}}=0$,

$$
\text { or, } \boldsymbol{k}_{\boldsymbol{i}}=\boldsymbol{c}_{\boldsymbol{i}}, \quad \boldsymbol{i}=1,2, \cdots \text {. }
$$

\section{The Method of Completing the Squares}

Resuming function

$$
\begin{aligned}
& Q\left(\boldsymbol{k}_{1}, \boldsymbol{k}_{2}, \cdots\right) \\
& =\|\boldsymbol{x}\|^{2}-2 \sum_{i=1}^{\infty} \boldsymbol{k}_{\boldsymbol{i}} \boldsymbol{c}_{\boldsymbol{i}}+\sum_{\boldsymbol{i}=1}^{\infty} \boldsymbol{k}_{\boldsymbol{i}}{ }^{2} \\
& =\|\boldsymbol{x}\|^{2}-\sum_{\boldsymbol{i}=1}^{\infty} \boldsymbol{c}_{\boldsymbol{i}}{ }^{2}+\sum_{\boldsymbol{i}=1}^{\infty} \boldsymbol{c}_{\boldsymbol{i}}{ }^{2}-2 \sum_{\boldsymbol{i}=1}^{\infty} \boldsymbol{k}_{\boldsymbol{i}} \boldsymbol{c}_{\boldsymbol{i}}+\sum_{\boldsymbol{i}=1}^{\infty} \boldsymbol{k}_{\boldsymbol{i}}{ }^{2} \\
& =\|\boldsymbol{x}\|^{2}-\sum_{\boldsymbol{i}=1}^{\infty} \boldsymbol{c}_{\boldsymbol{i}}{ }^{2}+\sum_{\boldsymbol{i}=1}^{\infty}\left(\boldsymbol{k}_{\boldsymbol{i}}-\boldsymbol{c}_{\boldsymbol{i}}\right)^{2} \\
& =\min \Leftrightarrow \\
& \boldsymbol{k}_{\boldsymbol{i}}=\boldsymbol{c}_{\boldsymbol{i}}, \quad \boldsymbol{i}=1,2, \cdots
\end{aligned}
$$

Hence, the minimum value of this type is

$$
\boldsymbol{Q}_{\min }\left(\boldsymbol{k}_{1}, \boldsymbol{k}_{2}, \cdots\right)=\|\boldsymbol{x}\|^{2}-\sum_{i=1}^{\infty} \boldsymbol{k}_{\boldsymbol{i}}{ }^{2} \text {. }
$$

Comparison of the above can be found in three forms: although the process of seeking the best are different, but the result was the same. By comparison we obtain: in theory, The method of projection must be based on Hilbert space theory, the theory used is high deep. In the method, the method of derivation is the most easily only need to have a viable method for the derivation can be. In theory, The method of completing the squares is belong to the scope of elementary algebra, as long as the analytic objective function is relatively simple, can be solved by elementary methods. In the results, with the best way to not only find the optimal coefficients $C_{i}$, and obtain the minimum value of the objective function is $\boldsymbol{Q} \min \left(\boldsymbol{k}_{1}, \boldsymbol{k}_{2}, \cdots\right)$. The following for Wiener filtering and system identification to explore for solving Least Squares Estimator in signal processing by using of the method of completing the squares.

III. THE APPLICATION OF THE METHOD OF COMPLETING THE SQUARES IN WIENER FILTER

Let $\boldsymbol{X}=\left[\boldsymbol{x}_{1}, \boldsymbol{x}_{2}, \cdots, \boldsymbol{x}_{n}\right]^{T}$ is a random vector.

It contains a real signal: $\boldsymbol{s}=\left[\boldsymbol{s}_{1}, \boldsymbol{s}_{2}, \cdots, \boldsymbol{s}_{n}\right]^{T}$ and Random noise: $\boldsymbol{v}=\left[\boldsymbol{v}_{1}, \boldsymbol{v}_{2}, \cdots, \boldsymbol{v}_{n}\right]^{T}$.

Let $\boldsymbol{H}$ is a known $\boldsymbol{n} \times \boldsymbol{n}$ interference matrix. Considering the following model: $\boldsymbol{x}=H \boldsymbol{s}+\boldsymbol{v}$ [5-7], now, we set $\boldsymbol{T}$ is an orthogonal transformation, $\boldsymbol{x}$ is inputted to the system shown in Figure 1.

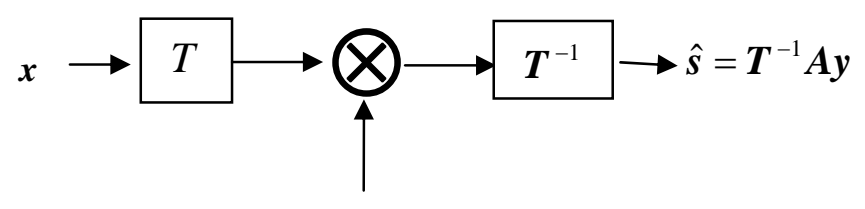

A

Figure 1 The block diagram of Wiener filter

For the corresponding output to be the best estimate of the true signal, that is the minimum of the mean square error, or,

$\boldsymbol{\varepsilon}=\|\boldsymbol{s}-\hat{\boldsymbol{s}}\|^{2}=E\left\{(\boldsymbol{s}-\hat{\boldsymbol{s}})^{T}(\boldsymbol{s}-\hat{\boldsymbol{s}})\right\}=\min$.

Then, Wiener filter matrix $A$ is calculated by the Method of Completing the Squares.

Here, According to Figure 1, we know that $\hat{\boldsymbol{s}}=T^{-1} A \boldsymbol{y}=T^{-1} A T \boldsymbol{x}$.

Therefore,

$$
\begin{aligned}
& \varepsilon=E\left\{(s-\hat{s})^{T}(s-\hat{s})\right\} \\
& =\operatorname{tr} E\left\{(s-\hat{s})^{T}(s-\hat{s})\right\} \\
& =\operatorname{tr} E\left\{\left(s-T^{-1} A y\right)\left(s-T^{-1} A y\right)^{T}\right\} \\
& =\operatorname{tr}\left[E\left\{s s^{T}\right\}+T^{-1} A E\left\{y y^{T}\right\} A^{T} T\right. \\
& \left.\quad-E\left\{s y^{T}\right\} A^{T} T-T^{-1} A E\left\{y s^{T}\right\}\right]
\end{aligned}
$$




$$
\begin{aligned}
& =\operatorname{tr}\left[\boldsymbol{E}\left\{\boldsymbol{s} \boldsymbol{s}^{T}\right\}\right]-\operatorname{tr}\left[\boldsymbol{E}\left\{\boldsymbol{s} \boldsymbol{y}^{T}\right\} \boldsymbol{E}\left\{\boldsymbol{y} \boldsymbol{y}^{T}\right\}^{-1} \boldsymbol{E}\left\{\boldsymbol{y} \boldsymbol{s}^{T}\right\}\right] \\
& +\operatorname{tr}\left[\left(T^{-1} A-E\left\{s y^{T}\right\} E\left\{y y^{T}\right\}^{-1} E\left\{y y^{T}\right\}\right.\right. \\
& \left.\times\left(\boldsymbol{T}^{-1} \boldsymbol{A}-\boldsymbol{E}\left\{\boldsymbol{s y ^ { T }}\right\} \boldsymbol{E}\left\{\boldsymbol{y y ^ { T }}\right\}^{-1}\right)^{T}\right] \\
& =\min \\
& \Leftrightarrow \boldsymbol{T}^{-1} \boldsymbol{A}=\boldsymbol{E}\left\{\boldsymbol{s} \boldsymbol{y}^{\boldsymbol{T}}\right\} \boldsymbol{E}\left\{\boldsymbol{y} \boldsymbol{y}^{\boldsymbol{T}}\right\}^{-1} .
\end{aligned}
$$

Solving above them, we find

$$
A=T E\left\{\boldsymbol{s} \boldsymbol{y}^{T}\right\} E\left\{\boldsymbol{y} \boldsymbol{y}^{T}\right\}^{-1}
$$

and we find the minimum error is

$$
\boldsymbol{\varepsilon}_{\min }=\operatorname{tr}\left[E\left\{\boldsymbol{s} \boldsymbol{s}^{T}\right\}\right]-\operatorname{tr}\left[E\left\{\boldsymbol{s} \boldsymbol{y}^{T}\right\} E\left\{\boldsymbol{y} \boldsymbol{y}^{T}\right\}^{-1} E\left\{\boldsymbol{y s}{ }^{T}\right\}\right] .
$$

Here, in order to simplified (2), we make the following statistical assumptions:

$$
\left\{\begin{array}{l}
E\{\boldsymbol{x}\}=0, \quad E\{\boldsymbol{y}\}=0 \\
E\left\{\boldsymbol{s} \boldsymbol{v}^{T}\right\}=E\left\{\boldsymbol{v} \boldsymbol{s}^{T}\right\}=0
\end{array},\right.
$$

and let the true signal $\boldsymbol{S}$ and noise $\boldsymbol{v}$ autocorrelation matrix to meet the need of

$$
\begin{aligned}
& E\left\{s \boldsymbol{s}^{T}\right\}=P, \quad E\left\{\boldsymbol{v} \boldsymbol{v}^{T}\right\}=R, \\
& \text { then, } \boldsymbol{E}\left\{\boldsymbol{s} \boldsymbol{y}^{\boldsymbol{T}}\right\}=\boldsymbol{E}\left\{\boldsymbol{s} \boldsymbol{x}^{\boldsymbol{T}}\right\} \boldsymbol{T}^{\boldsymbol{T}} \\
& \quad=\boldsymbol{E}\left\{\boldsymbol{s}(\boldsymbol{H} \boldsymbol{s}+\boldsymbol{v})^{\boldsymbol{T}}\right\} \boldsymbol{T}^{\boldsymbol{T}}=\boldsymbol{P} \boldsymbol{H}^{\boldsymbol{T}} \boldsymbol{T}^{\boldsymbol{T}} .
\end{aligned}
$$

Whence,

$$
E\left\{x x^{T}\right\}=E\left\{(H s+v)(H s+v)^{T}\right\}=H P H^{T}+R,
$$

and $\boldsymbol{E}\left\{y \boldsymbol{y}^{T}\right\}=\boldsymbol{T}\left(\boldsymbol{H P H} \boldsymbol{H}^{T}+\boldsymbol{R}\right) \boldsymbol{T}^{T}$.

Let (4), (5), (6) into (2) and (3), whence, we have Wiener filter matrix :

$$
\begin{aligned}
\boldsymbol{A} & =\boldsymbol{T} \boldsymbol{E}\left\{\boldsymbol{s y ^ { T }}\right\} \boldsymbol{E}\left\{y y^{T}\right\}^{-1} \\
& =\boldsymbol{T P H} \boldsymbol{H}^{T}\left(\boldsymbol{H P H} \boldsymbol{H}^{T}+\boldsymbol{R}\right)^{-1} \boldsymbol{T}^{T}
\end{aligned}
$$

and the minimum error :

$$
\begin{aligned}
& \varepsilon_{\min }=\operatorname{tr}\left[\boldsymbol{E}\left\{\boldsymbol{s s}{ }^{T}\right\}\right]-\operatorname{tr}\left[\boldsymbol{E}\left\{\boldsymbol{s} \boldsymbol{y}^{T}\right\} \boldsymbol{E}\left\{\boldsymbol{y} \boldsymbol{y}^{T}\right\}^{-1} \boldsymbol{E}\left\{\boldsymbol{y s ^ { T }}\right\}\right] \\
& =\operatorname{tr}\left[\boldsymbol{P}-\boldsymbol{P}\left(\boldsymbol{H P H} \boldsymbol{H}^{T}+\boldsymbol{R}\right) \boldsymbol{P}^{T}\right] .
\end{aligned}
$$

\section{THE APPLICATION OF THE METHOD OF COMPLETING THE SQUARES IN SYSTEM IDENTIFICATION, ,}

Considering the multiple input multiple output system identification problem [8-10]. Assuming that the input is $\boldsymbol{x}_{1}, \boldsymbol{x}_{2}, \cdots, \boldsymbol{x}_{\boldsymbol{n}}$, and that the output is $\mathrm{y}_{1}, \boldsymbol{y}_{2}, \cdots, \boldsymbol{y}_{\boldsymbol{p}}$,

The relationship between them is $y_{i}=a_{1 i} x_{1}+a_{2 i} x_{2}+\cdots+a_{n i} x_{n}, \quad i=1,2, \cdots, p$.

Or credited as $\boldsymbol{y}^{T}=\boldsymbol{x}^{T} \boldsymbol{A}$.

Here

$\boldsymbol{y}^{\boldsymbol{T}}=\left[\mathrm{y}_{1}, \boldsymbol{y}_{2}, \cdots, y_{p}\right]$,

$x^{T}=\left[x_{1}, x_{2}, \cdots, x_{n}\right]$,

$\boldsymbol{A}=\left[\begin{array}{cccc}\boldsymbol{a}_{11} & \boldsymbol{a}_{12} & \cdots & \boldsymbol{a}_{1 p} \\ \boldsymbol{a}_{21} & \boldsymbol{a}_{22} & \cdots & \boldsymbol{a}_{2 p} \\ \vdots & \vdots & \vdots & \vdots \\ \boldsymbol{a}_{\boldsymbol{n} 1} & \boldsymbol{a}_{\boldsymbol{n} 2} & \cdots & \boldsymbol{a}_{\boldsymbol{n} \boldsymbol{p}}\end{array}\right]$.

Set the input and the output of the first observation values are $\boldsymbol{x}^{\boldsymbol{T}}(\boldsymbol{k})$ and $\boldsymbol{y}^{\boldsymbol{T}}(\boldsymbol{k}), \boldsymbol{k}=1,2, \cdots, \boldsymbol{m}$, then the identification problem is: seek to meet

$$
\left[\begin{array}{c}
y^{T}(1) \\
y^{T}(2) \\
\vdots \\
y^{T}(m)
\end{array}\right]=\left[\begin{array}{c}
x^{T}(1) \\
x^{T}(2) \\
\vdots \\
x^{T}(m)
\end{array}\right] A
$$

Simple recorded as $\boldsymbol{Y}=\boldsymbol{X} \boldsymbol{A}$,

here ,

$\boldsymbol{Y}$ is an $\boldsymbol{m} \times \boldsymbol{p}$ matrix, $\boldsymbol{X}$ is an $\boldsymbol{m} \times \boldsymbol{n}$ matrix.

Thus, multiple input multiple output linear system identification problem description for an $n \times m$ matrix $A$, so as to meet the following non negative definite matrix to achieve minimum:

$$
\boldsymbol{J}(\boldsymbol{A})=(\boldsymbol{Y}-\boldsymbol{X A})^{\boldsymbol{T}}(\boldsymbol{Y}-\boldsymbol{X A})=\min .
$$

Solving by the Method of Completing the Squares, we have

$\boldsymbol{J}(\boldsymbol{A})=(\boldsymbol{Y}-\boldsymbol{X A})^{T}(\boldsymbol{Y}-\boldsymbol{X A})$

$=Y^{T} Y-A^{T} X^{T} Y-Y^{T} X A+A^{T} X^{T} X A$

$=\boldsymbol{Y}^{T} \boldsymbol{Y}-\boldsymbol{Y}^{T} \boldsymbol{X}\left(\boldsymbol{X}^{T} \boldsymbol{X}\right)^{-1} \boldsymbol{X}^{T} \boldsymbol{Y}$

$+\left[\boldsymbol{A}-\left(\boldsymbol{X}^{T} \boldsymbol{X}\right)^{-1} \boldsymbol{X}^{T} \boldsymbol{Y}\right]^{T}\left(\boldsymbol{X}^{T} \boldsymbol{X}\right)\left[\boldsymbol{A}-\left(\boldsymbol{X}^{T} \boldsymbol{X}\right)^{-1} \boldsymbol{X}^{T} \boldsymbol{Y}\right]$

$\min \Leftrightarrow A=\left(\boldsymbol{X}^{\boldsymbol{T}} \boldsymbol{X}\right)^{-1} \boldsymbol{X}^{\boldsymbol{T}} \boldsymbol{Y}$.

Here ,

we suppose $\boldsymbol{X}^{\boldsymbol{T}} \boldsymbol{X}$ is reversible. 
The method of completing the squares is a skill very strong method, it gives the objective function to achieve minimal sufficient and necessary conditions are given, and the method of derivation is only a necessary condition extremum. The flexible use of the method of completing the squares for solving least squares problems are worthy of our exploration.. Through the method of completing the squares, can simplify the expression, a series of transformation process, a greater flexibility, we are good at association and expansion. Through the method of completing the squares to find the known and the unknown contact, thus simplifying the complex. The flexible application of the method of completing the squares can make the least square problem more deeply.

\section{ACKNOWLEDGEMENTS}

This work is supported by Funding Project for Students scientific research programs in Beijing. (08150115/074)

Funding Project for Teaching key projects by Beijing Institute of Graphic Communication (22150115039) ,.

\section{REFERENCES}

[1] ZHANG Eryan, ZHU Xiaofeng. Application of Deducing Least Square Estimation by Making Clever Use of Method of Completing the Squares[J].Statistics and Decision, vol.13(2014), pp. 14 - 15. (In Chinese)

[2] YI Danhui. Statistical Forecast [M]. China Renmin University Press, Beijing, 1990.

[3] SHEN Dingxuan. Random Process [M]. Central University of Science and Technology University Press Wuchang, 1990.

[4] LIU Chongkan. Mathematical Modes of Signal Processing[M]. Southeast University Press, Nanjing,1992.

[5] WU Dazheng. Signal and Linear System Analysis[M]. Higher Education Press, Beijing,1986.

[6] XU Shufang. Theory and Methods of Matrix Computation[M]. Peking University Press, Beijing,1995.

[7] Deng Zili. Optimal Filtering Theory and Applications, Modern Time Series Analysis Method [M]. Harbin: Press of Harbin Institute of Technology, 2000.

[8] Ignagni M B. Separate-bias Kalman filter with bias state noise [J]. IEEE Trans. Automatic Control, vol. 35 (1990), No.1, pp. 338 - 341.

[9] Lü Xiaoguang, TAN Fei, JIANG Le. New Decomposition Formula for the Inversion of Toeplitz Matrices,J. Journal of Huaihai Institute of Technology (Natural Sciences Edition), vol. 16(2007), No.1, pp.13-15. (In Chinese)

[10] LIU Zuanwu. Discussion on the Toeplitz Cyclic Matrix,J. Journal of Institute of Surveying and Mapping, vol.19(2002), No.2 2, pp. 82-84. (In

Chinese) 